\title{
No Effects of Intra-Articular Injections of Sodium Hyaluronate, Corticosteroids, Platelet-Rich Plasma on Temporomandibular Joint Osteoarthritis: a Systematic Review and Network Meta-analysis of Randomized Controlled Trials
}

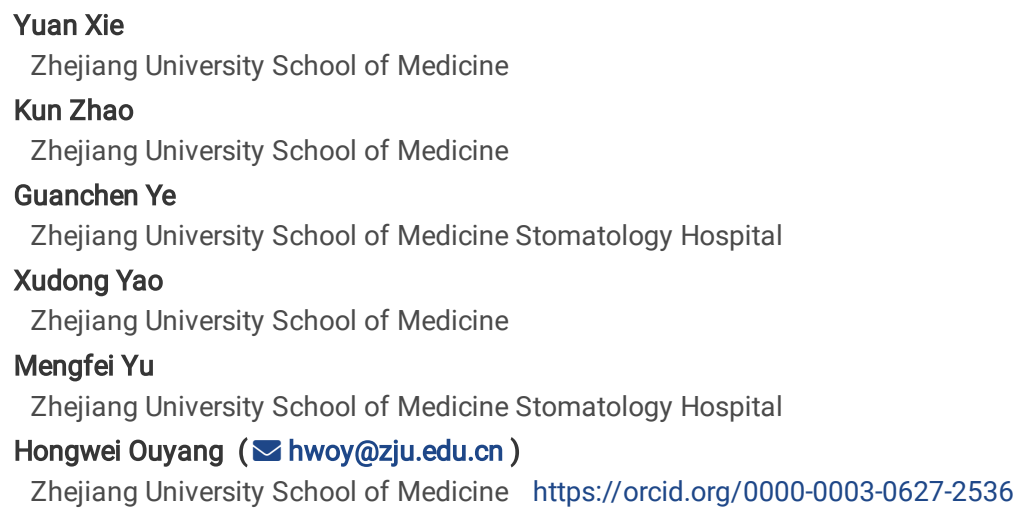




\section{Abstract \\ Background}

Intra-articular injections of corticosteroids (CCS), hyaluronic acid (HA), and platelet-rich plasma (PRP) have often been used for temporomandibular joint osteoarthritis (TMJ OA). However, there is no guideline for the choice of pharmacological injections. The aim of this network meta-analysis (NMA) is to compare the efficacy of different intra-articular injectable treatments on TMJ OA.

\section{Methods}

Studies were identified from PubMed, Embase and Cochrane Central with date up to December 2020. Randomized Controlled Trials (RCTs) included were the studies of patients with TMJ OA who had intra-articular treatment with CCS, HA, PRP, placebo and follow-up assessing TMJ function in target outcome variables. The primary outcome was temporomandibular joint pain (VAS). The secondary outcomes were maximal mouth opening (mm), and lateral movement to the affected side $(\mathrm{mm})$.

\section{Results}

Nine RCTs involving 316 patients were included. For primary pain outcome, no significance was detected when CCS, HA and PRP were compared with placebo by both short- (3-6 months) and long-term (>12 months) follow-up. In addition, these injectables did not significantly outperform placebo by evaluating secondary functional outcomes (maximal mouth opening and lateral movement) with the same follow-up. Subgroup analyses showed that the effect of CCS on subgroups with more than $70 \%$ women was statistically less effective compared with placebo.

\section{Conclusion}

Evidence suggested that intra-articular pharmacological injections of CCS, HA, and PRP had no effect on improving TMJ pain and functional outcomes compared with placebo injection.

\section{Trial registration}

This study is registered with PROSPERO, number CRD42021270914.

\section{Background}

The temporomandibular joint (TMJ), or jaw joint, is a synovial articulation that performs complicated everyday functions such as speech and mastication.(1) Temporomandibular disorders (TMDs), representing a range of conditions with multifactorial complex pathophysiology, are one of the most common causes of pain in the mouth and face and have the potential to produce chronic pain conditions. $(2,3)$ Temporomandibular joint dysfunction not only causes pain and disability in 20 to $25 \%$ of adults worldwide, but also huge economic costs estimated at \$4 billion per year.(4) Temporomandibular joint osteoarthritis (TMJ $\mathrm{OA})$, characterized by progressive cartilage degradation, remodeling of the condylar bone, and synovitis in addition to chronic pain,(5) is one of the TMDs.

Several treatment modalities of TMJ OA includes nonsurgical therapies such as physical therapies, occlusal splints, NSAIDs, and minimally invasive therapy of intra-articular injections with hyaluronic acid (HA), corticosteroids (CCS), or platelet-rich plasma (PRP). $(1,3,6)$ Injectable pharmacological treatments, which are more invasive than other topical or oral therapies, are often reserved for patients who have not responded favorably to most or all other nonsurgical treatment modalities and have mild to moderate OA.(7) Previous systematic studies have suggested intra-articular injection as a first-line therapy for TMJ OA, (8) particularly in pain control. ${ }^{(9)}$ However, there is no guideline for the choice of injection pharmaceutics.

While a previous NMA on knee OA suggested the superiority of intra-articular treatments over placebo, (10) a more recent NMA showed that intra-articular saline injections performed as well as any other injectable option in improving pain and functional outcomes in hip OA.(7) However, the efficacy and effectiveness of CCS, HA and PRP compared with placebo injections, as well as the rank probability as the best treatment among these three pharmacological treatments remains unkown in TMJ OA, in spite of the previous meta-analyses on the relevant topics.(11-13) Besides, osteoarthritis is universally acknowledged as an age-related and female-dominant condition, $(14,15)$ and our previous work showed that the efficacy of PRP injection treatment for OA was related to sample size and gender composition.(16) Consequently, it implicated the location-dependent and subgroup-dependent effects of intra-articular injectables in TMJ OA. With new RCTs comparing various IA injections on TMJ OA conducted since, it is of great importance to provide useful messages about pharmacological injection therapies and potential patient subgroup information for TMJ OA in the clinical context.

Thus, it warrants the need for a systematic review and network meta-analysis to determine: whether pharmacological injections of CCS, HA and PRP could outperform injection of placebo, and if so, the possible treatment effect ranking of the three pharmacological injections of TMJ OA. Also, we believe it is necessary to conduct subgroup analyses on age and gender composition for further investigations.

\section{Methods}




\section{Search strategy and protocols}

We searched PubMed, Embase and Cochrane Central Register of Controlled Trials from inception to December 2020 (Additional file 1). Only Randomized Controlled Trials (RCTs) for humans were included, with no language restriction. Additionally, reference lists of reviews on the relevant topic were manually scanned. This study follows the guidance of Cochrane Library and the Preferred Reporting Items for Systematic Reviews and Meta-Analyses (PRISMA) guidelines for conducting and reporting systematic reviews and network meta-analysis.(17)

\section{Eligibility criteria}

The PICO framework was employed as the critera for selection of RCTs:

Population (P): Adult patients who have been diagnosed as TMJ OA on the criteria of Research Diagnostic Criteria/ temporomandibular disorders (RDC/TMDs), Diagnostic Criteria/ temporomandibular disorders (DC/TMDs) (Axis I Group IIlb), or imaging findings that demonstrated mild to severe degenerative changes.

Intervention (I) and Comparison (C): Included RCTs should involve two or more intra-articular injections of the following injectables - corticosteroids (CCS), hyaluronic acid (HA), platelet-rich plasma (PRP), and placebo injections (Ringer's Lactated Solution). Small differences of dosage and injection intervals between RCTs were ignored. Included studies were not necessarily required to include placebo injection. Instead, studies that compare two or more injectables were also included.

Outcome (0): Included RCTs had to report the primary outcome pain (VAS scale), one or both of the secondary outcomes (maximal mouth opening and lateral movement to the affected side) with a follow-up of at least 3 months.

\section{Study selection}

Two blinded reviewers independently reviewed all articles at the 1) title/abstract and 2) full text stages. Any discrepancy in the title/abstract stage resulted in the article being forwarded directly to the full-text stage. At the full-text stage, any discrepancy was resolved by consensus.

\section{Data extraction}

Data from each study was extracted and reviewed for potential inconsistency by two extractors. Basic data included study characteristics, country(s) of recruited patients, age and gender, dropout(s), dose(s) and frequency of the intra-articular injection(s), whether prior arthrocentesis was performed, and other details of intervention(s). Quantitative data included pain as the primary outcome variable, and secondary outcome variables including maximal mouth opening and lateral movement indicating functional scores. Quantitative data were recorded accurately along with follow-up time of measurements.

\section{Quality assessment}

Two reviewers independently assessed the risk of bias under aspects of allocation concealment, random sequence generation, blinding of investigators and patients, blinding of outcome variables examiners, integrity of data, and selective reporting using the Cochrane risk-of-bias tool(18). Each aspect was rated as low, high or unclear risk of bias. Any disagreement was resolved by consensus.

\section{Measures of treatment effect and summary measures}

The Visual Analogue Scale (VAS, 0-10) for pain and the maximal mouth opening (millimeters, mm) were two commonly assessed outcomes in both research studies and clinics. Lateral movement (millimeters, $\mathrm{mm}$ ) to the affected side was also included as secondary outcome with MMO. VAS that was originally recorded on a 0-100 scale was converted to the standard 0-10 scale, with 0 indicating no pain and 10 indicating the worst imaginable pain.

The changes in the pain scores, maximal mouth opening and lateral movement were calculated, and a negative value represented pain relief, while a positive value stands for improvement in fuctional outcomes. When studies did not report mean change, these values were calculated as the arithmetic difference between baseline and follow-up. The calculation for SD was available for each study. Measurements of outcome variables, which were weighted by sample size, were performed according to the Cochrane Handbook methods; weighted mean scores were calculated at baseline, short-term, and long-term follow-up timepoints.(18)

Mean Differences between pharmacological injections and placebo injections were summarized using forest plots, along with 95\% Confidence Intervals $(95 \% \mathrm{Cl})$ for pairwise meta-analysis, $95 \%$ Credible Intervals (95\% Crl) for NMA, and SUCRA values.

\section{Network meta-analysis}

The network plot, which includes the number of each intervention, how often they were evaluated and the frequency of comparisons between treatment options, was first generated to visually illustrate the basic information of the NMA. The nodes represented treatments whose size was weighted according to the number of studies that examined the treatment, while the lines represented studies that compared the two connected nodes, with the thickness of the lines indicating the number of studies.

Next, we made the assumption of transitivity that there were no systematic differences between the available comparisons other than the treatments being compared, and thus performed an NMA.(19) We used Bayesian random-effects models for mixed multiple treatment comparisons. Results were presented, along with $95 \%$ central credible intervals (Crl), as mean difference effect sizes for pain, maximal mouth opening, and lateral movement to the affected side. Each outcome variable was evaluated at short- (3-6 months) and long-term (>12 months) timepoints. 
Forest plots, leaguetables, and Ranking diagrams (Rankograms) were created. The surface under the cumulative ranking curve (SUCRA) scores, which indicates the probability that a specific treatment ranks first for a given outcome, were reported. A SUCRA value of $100 \%$ means that the treatment is certainly the most effective in the whole network, while a value of $0 \%$ means that it is certainly the least effective. A higher SUCRA percentage indicates that the given treatment was more likely to rank first among all treatment options for a given outcome. A higher SUCRA indicates a better rank of the given intervention in the network. $(19,20)$ Plus, subgroup analyses on age and gender, and sensitivity analyses on arthrocentesis were performed.

Inconsistency was assessed using the node-splitting method, while heterogeneity was assessed using ANOHE method. The statistical package used for the pairwise meta-analysis was 'meta', and those for network meta-analysis were 'rjags' and 'gemtc', all built under R V.4.0.2.

\section{Results}

The search strategy we employed retrieved 234 related studies ( 99 duplicates included). After reviewing the titles/abstracts, 99 were excluded. The remaining 36 articles were retrieved for full-text review, of which 9 met the inclusion criteria for meta-analysis (Figure 1).(21-28) The characteristics of the selected studies were given in Table 1. All studies included the primary outcome of pain, uniformly scored on the scale of VAS (scales of 0-100 were converted to 0-10). For the secondary outcomes, 6 of the 8 studies included maximal mouth opening ( $\mathrm{mm}$ ), while 5 included lateral movement to the affected side (mm). All included trials were published between 2007 and 2019, involving a total of 316 participants. Of the 9 studies included, only 2 were conducted without arthrocentesis. $(21,28)$ The sample size for each study was calculated as the number of patients, rather than the number of affected joints, for consistency. Four studies examined short-term (3-6 months) outcome, while the other 5 only reported long-term ( $>12$ months) outcome. Two studies had three comparative groups, while the other seven studies had two (placebo was counted as an independent group, as were HA, CCS, and PRP). The age of patients recruited in each study ranged from 32.5 to 51.7 years (one study did not report the mean age); the proportion of women in each study ranged from $30-90 \%$. 
Table 1

Characteristics of studies included

\begin{tabular}{|c|c|c|c|c|c|c|c|c|c|c|}
\hline $\begin{array}{l}\text { Study, } \\
\text { year }\end{array}$ & $\begin{array}{l}\text { Study } \\
\text { design }\end{array}$ & country & $\begin{array}{l}\text { Total } \\
\text { Patients }\end{array}$ & $\begin{array}{l}\text { Female } \\
\%\end{array}$ & Mean age $( \pm S D)$ & $\begin{array}{l}\text { Treatment } \\
\text { groups(n) }\end{array}$ & $\begin{array}{l}\text { Number } \\
\text { of } \\
\text { injections } \\
\text { (interval) }\end{array}$ & $\begin{array}{l}\text { Dosage } \\
(\mathrm{mL})\end{array}$ & $\begin{array}{l}\text { Measurement } \\
\text { timepoint } \\
\text { (months) }\end{array}$ & Dropout \\
\hline \multirow{2}{*}{$\begin{array}{l}\text { Bjørnland et } \\
\text { al, } 2007\end{array}$} & \multirow[t]{2}{*}{ RCT } & \multirow[t]{2}{*}{ Norway } & \multirow[t]{2}{*}{40} & \multirow[t]{2}{*}{82} & HA: $53.4(12.9)$ & $\mathrm{HA}(\mathrm{n}=20)$ & \multirow[t]{2}{*}{$2(14 d)$} & \multirow[t]{2}{*}{$0.7-1$} & \multirow[t]{2}{*}{$0.5,1,6$} & \multirow[t]{2}{*}{ none } \\
\hline & & & & & CS: $50.0(13.3)$ & $\operatorname{CS}(n=20)$ & & & & \\
\hline \multirow{2}{*}{$\begin{array}{l}\text { Hegab, } \\
2015\end{array}$} & \multirow[t]{2}{*}{ RCT } & \multirow[t]{2}{*}{ Egypt } & \multirow[t]{2}{*}{50} & \multirow[t]{2}{*}{58} & PRP: 39 (4.975) & arth+PRP $(n=25)$ & \multirow[t]{2}{*}{$3(1 \mathrm{wk})$} & \multirow[t]{2}{*}{1} & \multirow[t]{2}{*}{$1,3,6,12$} & \multirow[t]{2}{*}{9} \\
\hline & & & & & HA: 38.2 (4.368) & arth+HA(n=25) & & & & \\
\hline \multirow{2}{*}{$\begin{array}{l}\text { Cömert et } \\
\text { al, } 2015\end{array}$} & \multirow[t]{2}{*}{$\mathrm{RCT}$} & \multirow[t]{2}{*}{ Turkey } & \multirow[t]{2}{*}{30} & \multirow[t]{2}{*}{90} & Placebo:35.08(14.84) & arth $(n=12)$ & \multirow[t]{2}{*}{$4(4 w k)$} & \multirow[t]{2}{*}{1} & \multirow[t]{2}{*}{12} & \multirow[t]{2}{*}{ none } \\
\hline & & & & & PRP: 32.22(14.32) & arth+PRP $(n=18)$ & & & & \\
\hline \multirow{2}{*}{$\begin{array}{l}\text { Cömert, } \\
2016\end{array}$} & \multirow[t]{2}{*}{$\mathrm{RCT}$} & \multirow[t]{2}{*}{ Turkey } & \multirow[t]{2}{*}{24} & \multirow[t]{2}{*}{88} & Placebo:35.08(14.84) & arth $(n=12)$ & \multirow[t]{2}{*}{ N/A } & 1 & 12 & none \\
\hline & & & & & CS: 32.58(9.58) & arth+CS $(n=12)$ & & & & \\
\hline Cömert, & RCT & Turkey & 31 & 81 & $30.48(13.04)$ & arth+HA(n=13) & $4(4 w k)$ & 1 & 12 & none \\
\hline $\begin{array}{l}\text { Güngörmüş, } \\
2016\end{array}$ & & & & & & arth+PRP $(n=18)$ & & & & \\
\hline Bouloux et & RCT & USA & 102 & 84 & CS: 39.6(18.4) & arth $+C S(n=31)$ & $\mathrm{N} / \mathrm{A}$ & 1 & 1,3 & 4 \\
\hline & & & & & HA: 44.3(17.2) & arth+HA(n=36) & & & & \\
\hline & & & & & Placebo: 51.8(17.2) & $\begin{array}{l}\text { arth+LR } \\
\text { injection }(n=31)\end{array}$ & & & & \\
\hline Gurung, & RCT & India & 20 & 30 & $18-60$ & arth $(n=10)$ & $\mathrm{N} / \mathrm{A}$ & 0.5 & 1,3 & none \\
\hline 2017 & & & & & & arth+HA(n=10) & & & & \\
\hline Bergstrand, & $\mathrm{RCT}$ & Norway & 40 & 81 & Placebo:55(14.5) & arth $(n=17)$ & $\mathrm{N} / \mathrm{A}$ & 1 & 6,48 & 3 \\
\hline & & & & & HA: 47(15.7) & arth+HA(n=20) & & & & \\
\hline $\begin{array}{l}\text { Gokçe } \\
\text { Kutuk, } 2019\end{array}$ & $\mathrm{RCT}$ & Turkey & 31 & 63 & PRP:36.4(8.2) & $\operatorname{PRP}(n=13)$ & 3 (1 mo) & 1 & $1,2,3$ & none \\
\hline & & & & & HA: $37.4(9.9)$ & $\mathrm{HA}(\mathrm{n}=12)$ & & & & \\
\hline & & & & & CS: 34.5(9.3) & $\operatorname{CS}(n=6)$ & & & & \\
\hline
\end{tabular}

\section{Results of pairwise meta-analysis}

All of the direct comparison evidenced at both short- and long- term was imported. The mean differences in all the comparisons with their corresponding $95 \%$ Cls were calculated using a random-effects model (Figure 2).

For the primary outcome pain, it was demonstrated that the injection of CCS was inferior to that of placebo (MD, 1.73 [95\% Cl, 0.37 to 3.09$])$, and no significance was detected between HA and placebo injection (MD, -0.07 [95\% $\mathrm{Cl},-1.90$ to 1.77]) in the short term. Also, the injection of PRP was found to be superior to that of $\mathrm{HA}(\mathrm{MD},-3.50$ [95\% $\mathrm{Cl},-4.51$ to -2.49$])$. Hence, PRP was suggested as the best injection treatment option while CCS was the poorest in our pairwise comparisons. PRP further had outperformed HA (MD, -1.64 [95\% Cl, -2.28 to -1.00]) in the long term, while other comparisons were no longer statistically significant.

For the secondary outcome, no significance was detected in any of the pairwise comparisons (Additional file 2).

\section{Results of network meta-analysis}

The network of all treatment comparisons analyzed by pain, maximal mouth opening, and lateral movement to the affected side during the longest follow-up timepoint of each study, was shown in Figure 3. As indicated by the thickness of the branches between nodes, comparisons between HA and placebo were numerically the highest, whereas those between CCS and PRP were the lowest. Overall, the individual treatment groups included intra-articular placebo injection with or without arthrocentesis $(n=70), \operatorname{HA}(n=136),(n=69), \operatorname{PRP}(n=56)$. 
The quality assessment of the included trials with risk of bias both within and between studies were done (Additional file 3). The heterogeneity assessment and inconsistency assessment of both long and short term timepoints were also completed (Additional file 4).

\section{Primary outcome - pain:}

None of the TMJ intra-articular injections showed significant improvement in pain by calculation using VAS scores via comparing with placebo injections at either 3-6 months (4 trials, 193 patients), or >12 months ( 5 trials, 133 patients) (Figure 4A). League tables showed no significant result for pairwise comparisons by means of network meta-analyses (Figure $4 \mathrm{~B}, \mathrm{C}$ ).

\section{Secondary outcome - maximal mouth opening (MMO), lateral movement to the affected side}

None of the intra-articular TMJ injections showed significant improvement in either MMO or lateral movement compared with placebo injections at either short or long term follow-up timepoints (Additional file 5).

\section{Ranking probabilities}

NMA allowed ranking of treatments. The probability of each treatment taking a particular rank was calculated by means of rank probability plots (Rankograms) (Additional file 6). In comparison, SUCRA values, given in Figure 3, accounted for uncertainty in relative effect estimates and relative ranking, whereas the original ranking method overlooked this. However, it should be emphasized that ranking of the SUCRA value only provided a possible prediction, which means that the context of treatment effects should be considered when deciding on a specific treatment.

For primary outcome pain, PRP had the largest probability to be the best injectable option in our network at both 3-6 months (SUCRA $=72 \%$ ) and $>12$ months (SUCRA $=74 \%$ ). Nevertheless, non-significance of the result produced in our NMA could not substantiate the SUCRA value as statistically valid ranking and clinical reference.

\section{Subgroup (gender, age) and sensitivity analysis}

Subgroup analyses which investigated the impact of age and gender of patients on primary outcome pain were conducted (Table 2). A percentage of female over $70 \%$ was defined as high proportion of female, while low proportion of female was defined as the opposite. Though statistically insignificant, an exactly reversed SUCRA value was shown, in which CCS works the best and placebo the poorest in studies with less than $70 \%$ of women, conversely in studies with more than $70 \%$ of women. The effect of corticosteroids on studies with more than $70 \%$ of women was the only pharmacological treatment statistically worse compared with placebo in all our investigations of subgroup analysis and comparisons overall (MD, 1.66 [95\% Crl, 0.163 to 3.03]). Strikingly, the mean differences of pain (VAS scale) of injectables compared with placebo were positive for female-rich studies (HA (MD, 0.486 [95\% Crl, -0.773 to 1.63]), PRP (MD, 0.735 [95\% Crl, -1.21 to 2.54])), but all turned negative for studies with less than $70 \%$ women (CCS (MD, -4.75 [95\% Crl, -10.5 to 0.975$]$ ), $\mathrm{HA}$ (MD, -1.59 [95\% Crl, -5.77 to 2.57$]$ ) and PRP (MD, -3.93 [95\% Crl, -9.01 to 1.16])). A mean age of 40 was the threshold we set for the subgroup analysis of age. Although insignificantly different, it suggested that CCS, HA and PRP showed effectiveness in patient groups with a mean age under 40 years old (CCS (MD, -1.91 [95\% Crl, -5.58 to 2.25]), HA (MD, -0.551 [95\% Crl, -3.58 to 2.68]), PRP (MD, -1.86 [95\% Crl, -5.26 to 1.86])). Also, patient groups with a mean age above 40 respond to CCS rather poorly (MD, 1.35 [95\% $\mathrm{Crl},-0.71$ to 3.48$])$.

Sensitivity analysis was performed by limiting the treatments to those that were combined with arthrocentesis, namely from the aforementioned 7 out of 9 studies, and no significant difference was found.

Table 2

Results of Subgroup Analysis of Primary Outcome Pain. Mean difference of pharmacological injections were compared with placebo. CCS, corticosteroids; HA, hyaluronic acid; Placebo, Ringer's Lactated Solution; PRP, platelet-rich plasma; SUCRA, surface under the cumulative ranking curves; NA, not applicable.

\begin{tabular}{|lllll|}
\hline $\begin{array}{l}\text { Mean Difference }(95 \% \text { Crl) } \\
\text { SUCRA Value }\end{array}$ & CCS & HA & Placebo & PRP \\
\hline Mean Age & & & \\
Above 40 yrs & $1.35(-0.71,3.48)$ & $-0.170(-1.59,1.42)$ & NA & NA \\
Under 40 yrs & 7 & 77 & 66 & NA \\
& $-1.91(-5.58,2.25)$ & $-0.551(-3.58,2.68)$ & NA & $-1.86(-5.26,1.86)$ \\
\hline Gender Proportion & 73 & 32 & 21 & 74 \\
Women $>70 \%$ & $1.66(0.163,3.03)$ & $0.486(-0.773,1.63)$ & NA & $0.735(-1.21,2.54)$ \\
\hline Women $<70 \%$ & 6 & 60 & 86 & 48 \\
& $-4.75(-10.5,0.975)$ & $-1.59(-5.77,2.57)$ & NA & $-3.93(-9.01,1.16)$ \\
\hline
\end{tabular}

\section{Disccusion}

Intra-articular injections of CCS, HA and PRP showed no significant short- or long-term improvement in pain and function outcomes compared with placebo injection in TMJ OA patients. Interestingly, we found the the effect of CCS on subgroup with more than $70 \%$ women was statistically less effective compared 
with placebo.

As opposed to the findings that intra-articular pharmacological injections in knee OA are of significant treatment effects,(10) the NMA we conducted found the effects the three different types of injectables to be non-significant in all outcome variables, which shares similarities with the study of injections in hip OA.(7) Nonetheless, there are 3 possibilities that may shed light on reasons behind the non-significant effect of CCS, HA and PRP compared with placebo:

Firstly, emerging evidence indicates that the apparent efficacy of pharmacological intra-articular injection therapy is largely due to other factors, particularly either the placebo effect or the combined effect of arthrocentesis.(29) On one hand, the intrinsic effect of Ringer's lactate solution functions as a washout of inflammatory cells, which are associated with pain signaling and osseous change, and release of the stuck disc phenomenon, $(6,14)$ thereby alleviating the symptoms of TMJ OA. The function of Ringer's lactate solution potentially accounts for the positive treatment effects of the placebo in our NMA and the consequent lack of competence between pharmacological and placebo injections. On the other hand, the sensitivity analysis of arthrocentesis did not indicate any combined treatment effect of injectables with arthrocentesis. Nevertheless, it could be owing to the fact that 7 studies were done with arthrocentesis while 2 studies without, so that the effect of arthrocentesis is not strong enough to break the non-significance of the result.

Secondly, compared with the NMA of knee joint and hip joint OA, our study revealed a non-significant result similar with that of the hip joint OA, while Bannuru et al. found that all the injectables outperformed placebo in knee join OA, with HA being the most prabable to be effective in alleviating pain and improving joint function.(10) It seems that the location-dependent effect of intra-articuar injection is due to the anatomical difference: knee joint has much more synovial tissues than hip joint and TMJ joint. Synovium, a complex structure in knee joint but not in either hip joint of TMJ, could provide a potential explanation for the results. Specifically, the anti-inflammatory injectable corticosteroids could suppress synovitis in OA joint. Plus, the synovium contains a number of targets for pharmacological treatments, including steroid hormone receptor positive cells and senescent cells. $(30,31)$ Besides, HA is an intrinsic component of synovial fluid, and PRP could be bioactive factors to promote the function of synovium tissues for pain relief and cartilage repair.(32)

Thirdly, the subgroup analysis of our NMA indicated that the effect is subgroup-dependent, particularly defined by age and gender. The diversity of patients probably leads to the non-significance of all of the three injectables investigated in our study. This reminds us of the value of Individual Patient Data (IPD) Meta-analysis that examine patients individually, as noted in an NMA that some individuals with knee OA respond more robustly to the injections of HA than others,(33) which could also be performed in TMJ OA in the future. Thus, it is not accurate to define pharmacological injection treatment as contrary to the goal of cost-effective health care.

Subgroup analyses on age and gender included in our NMA indicated that female and elderly patients respond poorly to CCS intra-articular injection treatment. Injectables particularly CCS should be given with caution in the clinics. The proposed mechanism explaining the different effect of CCS - not only injections targeting muscle and joint, but also oral, inhaled, topical use targeting other diseases - on subgroups of patients is absent in current literature, which predicted future trends to encourage more clinical trials with large sample size to substantiate the effect of CCS on different age groups, and gender difference. Additionally, as suggested in 2015 by Lochbühler et al, intra-articular injections could reduce mandibular growth in adolescent patients. (34, 35) Taken all together, despite that there is more likelihood for pharmacological injections to benefit younger age groups for pain relief, the potential adverse effects could not be overlooked. Clinicians should be aware of the precisive choice of injectables based on the subgroup-dependent benefits and side effects.

Our study is equipped with strengths and advantages among systematic review of related topics. Liu et al. did a meta-analysis by comparing multiple injection options for TMJ OA, but their evaluation was at short-term, mostly 1-month follow-up results.(12) They found that PDGF was more effective in relieving pain and improving MMO, while HA was more effective in enhancing MMO. PRP ranked as the best treatment in alleviating pain, but our study revealed that this recommendation lacked significance. We also demonstrated that the mean difference of HA in improving MMO was positive in the short term but turned negative in the long term, also insignificantly. With the new RCTs published since, $(27,28)$ results of the effectiveness of pharmacological injection were updated, which extended the work of Liu et al. Additionally, the findings of subgroup analysis of age and gender in our NMA further implicated the targeted efficacy of injectable drugs, which previous meta-analyses omitted. There have been systematic reviews with qualitative descriptions on comparing the intraarticular injections for TMJ OA with a follow-up of more than 6 months,(13) but the qualitative opinions had the disadvantage of lacking statistical validation. Pairwise comparisons in our study demonstrated that PRP outperformed HA when outcome pain was considered, conforming to the the findings of Liapaki et al.(13) However, the network meta-analysis, with which we used the extracted data to the full, further confirmed that no pharmacological injection was found to be significantly superior to placebo with regards to pain.

Our NMA has several limitations. Firstly, the number and size of RCTs included were limited, which may introduce bias due to small study effects. Secondly, heterogeneity between studies could not be ignored in our NMA. Thirdly, language bias could be a concern, as we only included English language trials in our NMA. Fourthly, stratification of drug (e.g. low and high molecular weight (LMW / HMW) HA)(36) was insufficient in the RCTs included, which may bring confounding factors to our NMA.

\section{Conclusion}

We found that the intra-articular injections for TMJ OA showed no significant improvement in all but exhibited subgroup dependent effects. Further large RCTs are needed to confirm our results and to identify the subgroups of patients who might benefit from the specific injectables.

\section{Abbreviations}

TMJ, temporomandibular joint

OA, osteoarthritis 
NMA, network meta-analysis

$\mathrm{RCT}$, randomized controlled trial

VAS, visual analogue scale

$\mathrm{MD}$, mean difference

CCS, corticosteroids

$\mathrm{HA}$, hyaluronic acid

Placebo, Ringer's Lactated Solution

PRP, platelet-rich plasma

SUCRA, surface under the cumulative ranking curves

$\mathrm{Cl}$, confidence intervals

Crl, credible intervals

\section{Declarations}

\section{Ethics approval and consent to participate}

Not applicable.

\section{Consent for publication}

Not applicable.

\section{Availability of data and materials}

All data generated and analyzed during this study are included in this published article.

\section{Competing interests}

The authors declare that they have no competing interests.

\section{Funding}

This work was supported by the National Natural Science Foundation of China (81630065). The foundation had no role in the planning, conduct or reporting of this work.

\section{Authors' contributions}

YX and KZ contributed to conception and design. YX, KZ and GY contributed to acquisition, analysis, and interpretation, while MY and HO contributed to analysis and interpretation. $Y X, K Z, G Y$ and $X Y$ drafted the manuscript, while $M Y$ and $\mathrm{HO}$ critically revised the manuscript. All authors read and approved the final manuscript.

\section{Acknowledgements}

Not applicable.

\section{References}

1. Wang XD, Zhang JN, Gan YH, Zhou YH. Current Understanding of Pathogenesis and Treatment of TMJ Osteoarthritis. Journal of Dental Research. 2015;94(5):666-73.

2. Durham J, Newton-John TR, Zakrzewska JM. Temporomandibular disorders. Bmj. 2015;350:h1154.

3. James Hupp MT, Edward Ellis. Contemporary Oral and Maxillofacial Surgery. 7th ed. Louis, Mo: Mosby Elsevier; 2018 27th September 2018.721 p.

4. Vapniarsky N, Huwe LW, Arzi B, Houghton MK, Wong ME, Wilson JW, et al. Tissue engineering toward temporomandibular joint disc regeneration. Sci Transl Med. 2018;10(446).

5. Lee YH, Park HK, Auh QS, Nah H, Lee JS, Moon HJ, et al. Emerging Potential of Exosomes in Regenerative Medicine for Temporomandibular Joint Osteoarthritis. Int J Mol Sci. 2020;21(4). 
6. de Souza RF, Lovato da Silva CH, Nasser M, Fedorowicz Z, Al-Muharraqi MA. Interventions for managing temporomandibular joint osteoarthritis. Cochrane Database of Systematic Reviews. 2012(4).

7. Gazendam A, Ekhtiari S, Bozzo A, Phillips M, Bhandari M. Intra-articular saline injection as effective as corticosteroids, platelet-rich plasma and hyaluronic acid for hip osteoarthritis pain: a systematic review and network meta-analysis of randomised controlled trials. Br J Sports Med. 2020.

8. Al-Moraissi EA, Wolford LM, Ellis E, 3rd, Neff A. The hierarchy of different treatments for arthrogenous temporomandibular disorders: A network metaanalysis of randomized clinical trials. J Craniomaxillofac Surg. 2020;48(1):9-23.

9. Guarda-Nardini L, Masiero S, Marioni G. Conservative treatment of temporomandibular joint osteoarthrosis: intra-articular injection of sodium hyaluronate. J Oral Rehabil. 2005;32(10):729-34.

10. Bannuru RR, Schmid CH, Kent DM, Vaysbrot EE, Wong JB, McAlindon TE. Comparative effectiveness of pharmacologic interventions for knee osteoarthritis: a systematic review and network meta-analysis. Ann Intern Med. 2015;162(1):46-54.

11. Liu Y, Wu J, Fei W, Cen X, Xiong Y, Wang S, et al. Is There a Difference in Intra-Articular Injections of Corticosteroids, Hyaluronate, or Placebo for Temporomandibular Osteoarthritis? J Oral Maxillofac Surg. 2018;76(3):504-14.

12. Liu Y, Wu JS, Tang YL, Tang YJ, Fei W, Liang XH. Multiple Treatment Meta-Analysis of Intra-Articular Injection for Temporomandibular Osteoarthritis. J Oral Maxillofac Surg. 2020;78(3):373.e1-.e18.

13. Liapaki A, Thamm JR, Ha S, Monteiro J, McCain JP, Troulis MJ, et al. Is there a difference in treatment effect of different intra-articular drugs for temporomandibular joint osteoarthritis? A systematic review of randomized controlled trials. Int J Oral Maxillofac Surg. 2021.

14. Song H, Lee JY, Huh K-H, Park JW. Long-term Changes of Temporomandibular Joint Osteoarthritis on Computed Tomography. Scientific Reports. 2020;10(1):6731.

15. Alexiou K, Stamatakis H, Tsiklakis K. Evaluation of the severity of temporomandibular joint osteoarthritic changes related to age using cone beam computed tomography. Dentomaxillofac Radiol. 2009;38(3):141-7.

16. Zhao K, Liu YS, Nie LY, Qian LN, Nie NF, Leptihn S, et al. The influence of sample size and gender composition on the meta-analysis conclusion of platelet-rich plasma treatment for osteoarthritis. J Orthop Translat. 2020;22:34-42.

17. Hutton B, Salanti G, Caldwell DM, Chaimani A, Schmid CH, Cameron C, et al. The PRISMA Extension Statement for Reporting of Systematic Reviews Incorporating Network Meta-analyses of Health Care Interventions: Checklist and Explanations. Annals of Internal Medicine. 2015;162(11):777-84.

18. Higgins JPT ea. Cochrane Handbook for Systematic Reviews of Interventions, Version 6.2. The Cochrane Collaboration. 2021.

19. Rouse B, Chaimani A, Li T. Network meta-analysis: an introduction for clinicians. Intern Emerg Med. 2017;12(1):103-11.

20. Salanti G, Ades AE, loannidis JP. Graphical methods and numerical summaries for presenting results from multiple-treatment meta-analysis: an overview and tutorial. J Clin Epidemiol. 2011;64(2):163-71.

21. Bjørnland T, Gjaerum AA, Møystad A. Osteoarthritis of the temporomandibular joint: an evaluation of the effects and complications of corticosteroid injection compared with injection with sodium hyaluronate. Journal of oral rehabilitation. 2007;34(8):583-9.

22. Cömert Kiliç S, Güngörmüş M, Sümbüllü MA. Is Arthrocentesis Plus Platelet-Rich Plasma Superior to Arthrocentesis Alone in the Treatment of Temporomandibular Joint Osteoarthritis? A Randomized Clinical Trial. Journal of oral and maxillofacial surgery. 2015;73(8):1473-83.

23. Cömert Kiliç S. Does Injection of Corticosteroid After Arthrocentesis Improve Outcomes of Temporomandibular Joint Osteoarthritis? A Randomized Clinical Trial. Journal of oral and maxillofacial surgery. 2016;74(11):2151-8.

24. Cömert Kiliç S, Güngörmüş M. Is arthrocentesis plus platelet-rich plasma superior to arthrocentesis plus hyaluronic acid for the treatment of temporomandibular joint osteoarthritis: a randomized clinical trial. International journal of oral and maxillofacial surgery. 2016;45(12):1538-44.

25. Bouloux GF, Chou J, Krishnan D, Aghaloo T, Kahenasa N, Smith JA, et al. Is Hyaluronic Acid or Corticosteroid Superior to Lactated Ringer Solution in the Short-Term Reduction of Temporomandibular Joint Pain After Arthrocentesis? Part 1. J Oral Maxillofac Surg. 2017;75(1):52-62.

26. Gurung T, Singh RK, Mohammad S, Pal US, Mahdi AA, Kumar M. Efficacy of arthrocentesis versus arthrocentesis with sodium hyaluronic acid in temporomandibular joint osteoarthritis: A comparison. Natl J Maxillofac Surg. 2017;8(1):41-9.

27. Bergstrand S, Ingstad HK, Møystad A, Bjørnland T. Long-term effectiveness of arthrocentesis with and without hyaluronic acid injection for treatment of temporomandibular joint osteoarthritis. Journal of oral science. 2019;61(1):82-8.

28. Gokçe Kutuk S, Gökçe G, Arslan M, Özkan Y, Kütük M, Kursat Arikan O. Clinical and Radiological Comparison of Effects of Platelet-Rich Plasma, Hyaluronic Acid, and Corticosteroid Injections on Temporomandibular Joint Osteoarthritis. Journal of craniofacial surgery. 2019;30(4):1144-8. 
29. Jones IA, Togashi R, Wilson ML, Heckmann N, Vangsness CT, Jr. Intra-articular treatment options for knee osteoarthritis. Nat Rev Rheumatol. 2019;15(2):77-90.

30. van der Goes MC, Straub RH, Wenting MJ, Capellino S, Jacobs JW, Jahangier ZN, et al. Intra-articular glucocorticoid injections decrease the number of steroid hormone receptor positive cells in synovial tissue of patients with persistent knee arthritis. Ann Rheum Dis. 2012;71(9):1552-8.

31. Jeon $\mathrm{OH}, \mathrm{Kim}$ C, Laberge RM, Demaria M, Rathod S, Vasserot AP, et al. Local clearance of senescent cells attenuates the development of post-traumatic osteoarthritis and creates a pro-regenerative environment. Nat Med. 2017;23(6):775-81.

32. Wathier M, Lakin BA, Cooper BG, Bansal PN, Bendele AM, Entezari V, et al. A synthetic polymeric biolubricant imparts chondroprotection in a rat meniscal tear model. Biomaterials. 2018;182:13-20.

33. Trojian TH, Concoff AL, Joy SM, Hatzenbuehler JR, Saulsberry WJ, Coleman CI. AMSSM scientific statement concerning viscosupplementation injections for knee osteoarthritis: importance for individual patient outcomes. British Journal of Sports Medicine. 2016;50(2):84.

34. Stoustrup P, Twilt M. Therapy. Intra-articular steroids for TMJ arthritis-caution needed. Nat Rev Rheumatol. 2015;11(10):566-7.

35. Lochbühler N, Saurenmann RK, Müller L, Kellenberger CJ. Magnetic Resonance Imaging Assessment of Temporomandibular Joint Involvement and Mandibular Growth Following Corticosteroid Injection in Juvenile Idiopathic Arthritis. J Rheumatol. 2015;42(8):1514-22.

36. Iturriaga V, Vásquez B, Bornhardt T, Del Sol M. Effects of low and high molecular weight hyaluronic acid on the osteoarthritic temporomandibular joint in rabbit. Clin Oral Investig. 2021.

\section{Figures}
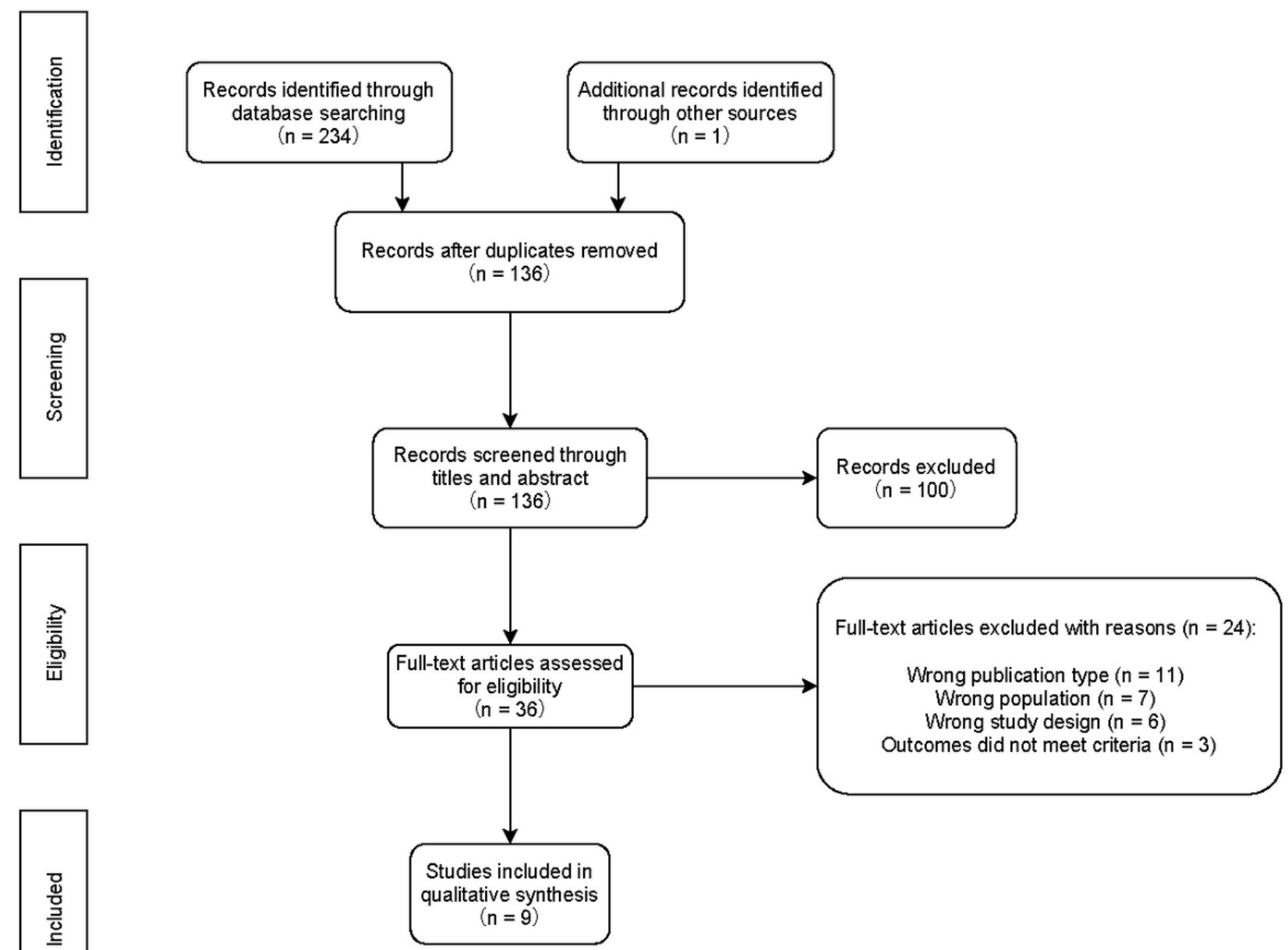

\section{Figure 1}

Flow chart of study selection. 


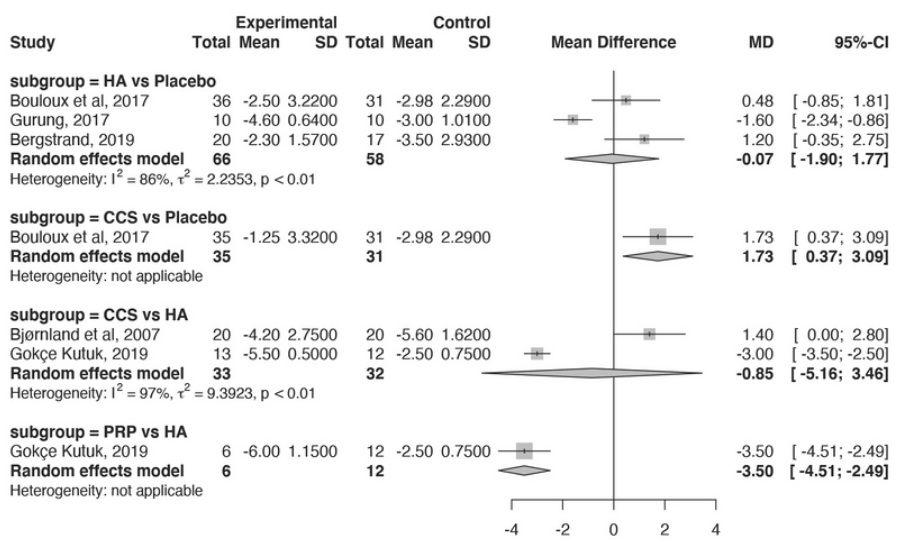

A

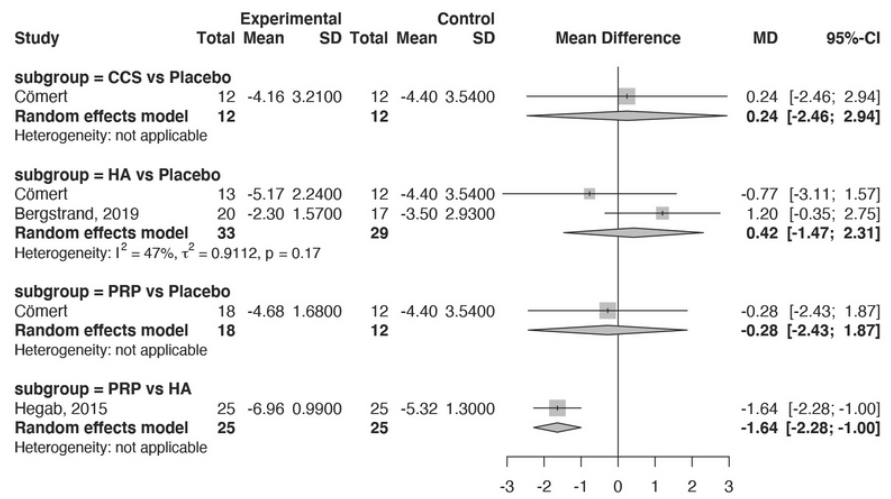

B

\section{Figure 2}

Forest plots demonstrating pairwise comparisons between CCS (corticosteroids), HA (hyaluronic acid), PRP (platelet-rich plasma) and placebo at both shortterm (A) and long-term (B) follow-up timepoints. 
A

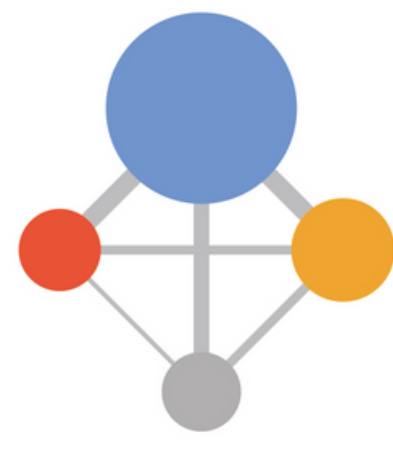

B

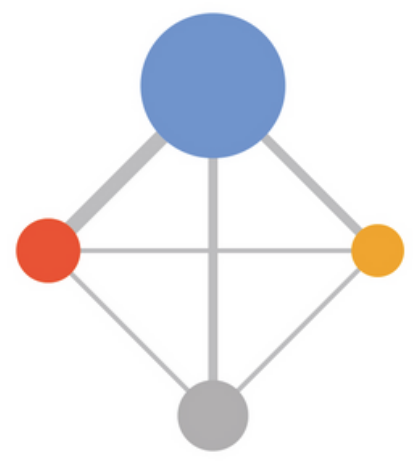

C

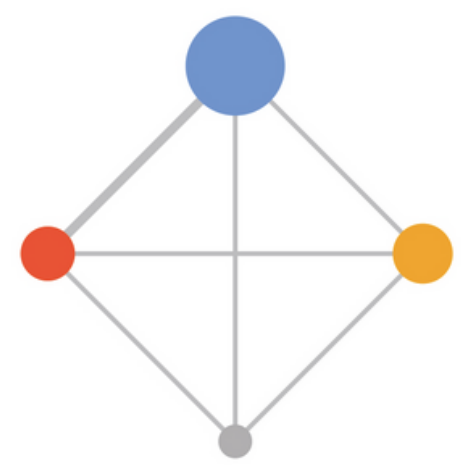

HA

CCS

PRP

Placebo

Figure 3

Overall network diagram for all treatment comparisons for outcomes of (A) pain (VAS scores), (B) maximal mouth opening, and (C) lateral movement to the affected side. The size of the nodes indicates the number of RCTs and sample size concerning the injectables, while the thickness of the sticks linking the nodes indicates the number of comparisons between injectables. CCS, corticosteroids; HA, hyaluronic acid; PRP, platelet-rich plasma. 


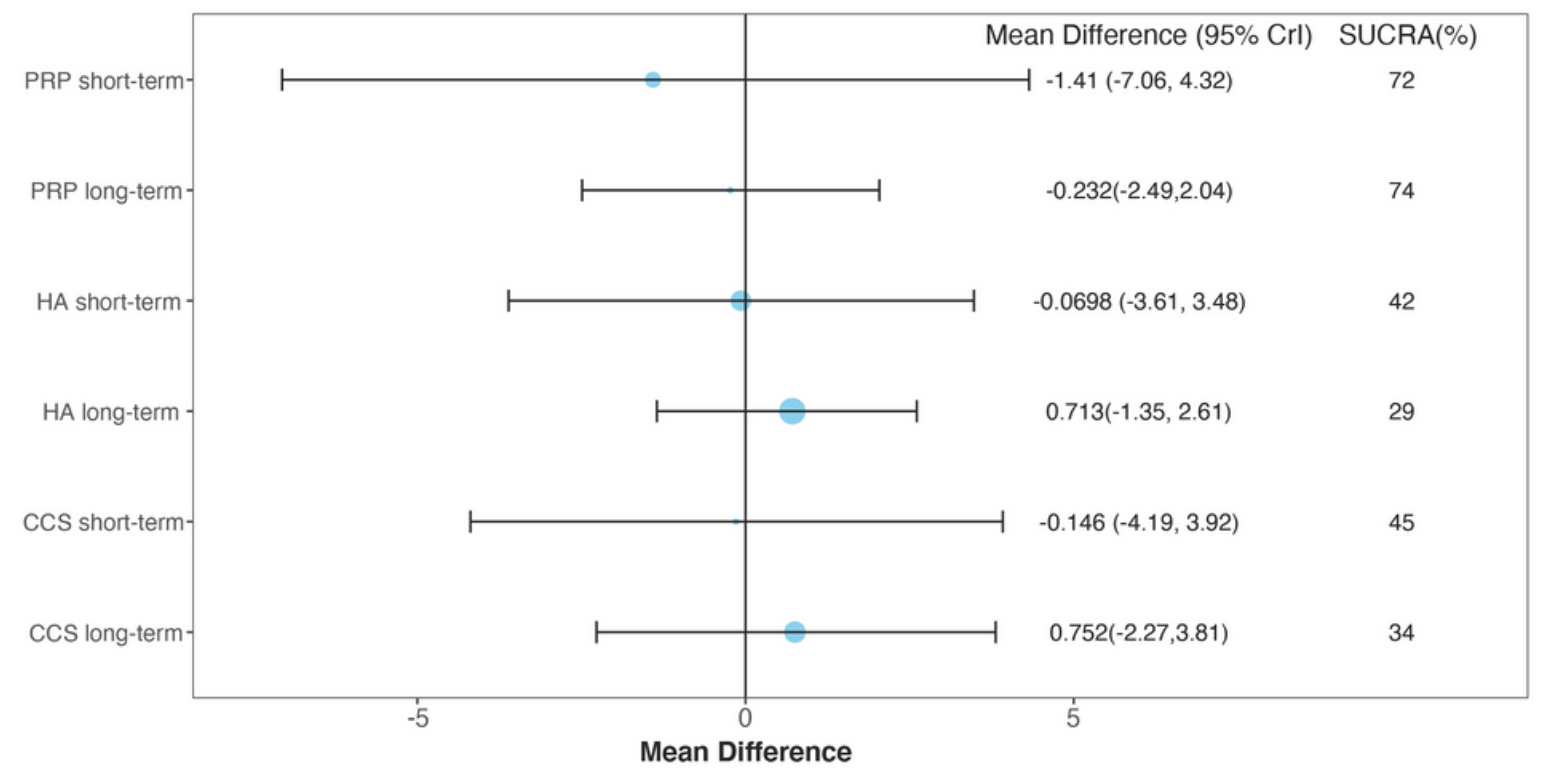

A

\begin{tabular}{|c|c|c|c|}
\hline CCS & & & \\
\hline$-0.07(-3.04,2.93)$ & $\mathrm{HA}$ & & \\
\hline$-0.15(-4.19,3.92)$ & $-0.07(-3.61,3.48)$ & Placebo & \\
\hline $1.27(-3.50,6.00)$ & $1.34(-3.41,6.05)$ & $1.41(-4.32,7.06)$ & PRF \\
\hline
\end{tabular}

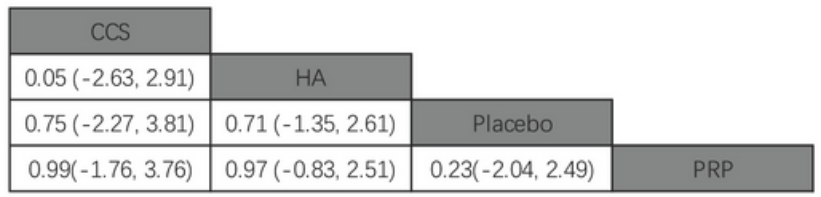

B

C

\section{Figure 4}

(A) Forest plot diagram showing the mean difference for changes in pain (VAS scores) at both short-term and long-term follow-up. A mean difference above zero indicates that treatment effects favor placebo injections, while a mean difference below zero means the opposite. (B, C) League tables demonstrating the results of the network meta-analyses comparing the reduction in VAS pain scores (95\% credible intervals) for short-term (B) and long-term (C) follow-up. Mean differences lower than 0 favour the column-defining treatment.

\section{Supplementary Files}

This is a list of supplementary files associated with this preprint. Click to download.

- Additionalfile1.docx

- Additionalfile2.docx

- Additionalfile3.docx

- Additionalfile4.docx

- Additionalfile5.docx

- Additionalfile6.docx 